\title{
A Thresholding Scheme of Eliminating False Detections on Vehicles in Wide-Area Aerial Imagery
}

\author{
Xin Gao* \\ Department of Electrical and Computer Engineering, \\ The University of Arizona, Tucson, AZ, USA \\ Email: tzgaoxin2016@ hotmail.com \\ *Corresponding author
}

\begin{abstract}
Post-processings are usually necessary to reduce false detections on vehicles in wide-area aerial imagery. In order to improve the performance of vehicle detection, we propose a two-stage scheme, which consists of a thresholding method by constructing a pixel-weight based thresholding policy to classify pixels in the grayscale feature map of an automatic detection algorithm followed by morphological filtering. We use two aerial videos for performance evaluation, and compare the automatic detection results with the ground-truth objects. We compute average F-score and percentage of wrong classifications towards six detection algorithms before and after applying the proposed scheme. We measure the variation of overlap ratios from detections to objects, and establish sensitivity analysis to evaluate the performance of proposed scheme by combining it on each of two representative algorithms. Simulation results verify both validity and efficiency of the proposed thresholding scheme, also display the difference of detection performance between datasets and among algorithms.
\end{abstract}

Keywords: Vehicle detection, thresholding, false positive, wide-area aerial imagery

Reference to this paper is made as follows: Author. Gao, X. (2018) 'A thresholding scheme of eliminating false detections on vehicles in wide-area aerial imagery', Int. J. Signal and Image Systems Engineering, Vol. X, No, X, pp. XX-XX.

Biographical notes: Xin Gao is pursuing Ph.D. degree in Department of Electrical and Computer Engineering with Ph.D. minor in Mathematics at the University of Arizona, Tucson, AZ, USA. Mr. Gao's research interests mainly include communications and coding theory, statistical signal processing, algorithms on digital image analysis and digital image processing, object detection and classification, data (signal, image and video, etc.) compression in intelligent transportation systems, bio medical signal processing and imaging devices, and dynamic quantitative fiscal modelling. He has already published more than 20 English research articles with Google scholar citations of over 240 times during his graduate study till early 2018 .

Copyright (C) 201x Inderscience Enterprises Ltd. 


\section{Introduction}

Detecting multiple ground vehicles from low-resolution aerial images stands for a fundamental challenging task on visual surveillance in wide-area aerial imagery [1], [6], [16]-[18]. The first step to process aerial frames often requires obtaining a normalized grayscale feature map, which also serves as the raw material for classifying true objects from backgrounds. Simply choosing a normalized grayscale pixel value or using a binary image thresholding algorithm to classify the feature map may bring up with high rate of false detections. Therefore, improving the performance of an automatic vehicle detection algorithm, often relies on an efficient post-processing scheme.

We discovered some shortcomings when applying a few existing post-processing schemes to our low-resolution wide-area aerial images: the dilation operation of binary masking [2] expands the size of correct detection; heuristic filtering [3] displays validity on reducing false detections only if vehicles have a uniformly distinct aspect ratio; filtering by shape index [4] yields limited improvement of average F-scores for each algorithm; the strategy of sieving and opening [5] indicates that some objects which actually exist, tend to be wrongly excluded if they are too close to other background targets which displays similar intensity.

For the purpose of achieving better classifications results, we intend to develop an efficient thresholding scheme to eliminate false positives on vehicle detection. In this paper, we construct a two-dimensional rectangle chessboard in which the square checkers are used to simulate each pixel in a grayscale image, and the normalized grayscale value of each pixel is calculated by averaging the weight from its four vertices. We employ the thresholding chessboard to justify each pixel on feature map whether it belongs to foreground or background regions, then classify the pixel belongings using the 8-connected component analysis. Morphological filters with best overall size of structuring elements, are designed to shape detections and eliminate tiny false detections after thresholding. We measure the performance improvement on the proposed thresholding scheme as sociated with six object detection algorith ms, and evaluate the robustness of our approach via a sensitivity analysis of the selected two algorithms combining with our scheme on overlap ratios from detections to objects.

\section{Datasets}

Two low-resolution aerial datasets $(720 \times 480$ pixels in spatial resolution for each frame) have been used for performance evaluation [16] of detection schemes with and without the proposed scheme. Traffic lanes were manually cropped from the aerial videos since our study was focused on vehicle detection. Note that either GIS mapping or utilizing an automatic detection method, can also perform the same task. Vehicles of rectangular shape represent the ground truth in our manual segmentation. For vehicles in Tucson dataset, the area is distributed from 40 to 150 pixels, and the total number of vehicles is 4012; in Phoenix dataset, vehicle area ranges from 20 to 175 pixels, and the total count is 4060 .

\section{The Proposed Scheme}

For pre-processing, we normalize the grayscale feature map obtained from applying a detection algorithm on each aerial frame, then perform the first stage of thresholding scheme by simulating a pixel-weighted thresholding chessboard to classify pixels on the grayscale feature map. This scheme is constructed in the following scenarios: each pixel can be simu lated as similar as the corresponding square checker on a thresholding chessboard, where each of the normalized grayscale pixel values must be weighted by averaging the "pattern weight" from its corresponding four vertices. Three typical layouts on the vertices of a thresholding chessboard are used to initialize the weight of vertices on the chessboard: black stones or white stones are weighted by 0 or 1 , and the empty cross-points without any stone are weighted by $1 / 2$.

Take the ground-truth objects for investigation. Objects in the normalized grayscale histogram can be either foreground or background dominated, or approximately equally distributed. The proposed thresholding scheme is used to classify pixels as foreground or background from a normalized grayscale feature map (with identical image size as the original grayscale frame), where a labelling policy of our thresholding scheme for a sample dataset (i.e., objects are foreground dominated) is derived as follows: 


\section{A Thresholding Scheme of Eliminating False Detections on Vehicles in Wide-Area Aerial Imagery}

Step 1: if the normalized grayscale value of one pixel is above the weight (3/4) of the pixel in the same position of thresholding chessboard (at least 3 white stones of the four vertices for this pixel), label it as a foreground pixel;

Step 2: if the normalized grayscale value of one pixel is below the weight (1/4) in the same position of thresholding chessboard (weighted by at least 3 black stones of its four vertices), label it as a background pixel;

Step 3: if the pixel weight is within the normalized grayscale threshold of [1/4, 3/4], we consider three cases:

Case 1: if the sum of this pixel and its 4 neighbourhood pixels weighs above $7 / 2$ (expected mean from $[1 / 4,3 / 4)+4$ * $3 / 4=7 / 2$ ), then label it as a foreground pixel;

Case 2: if the sum of this pixel and its 4 neighbourhood pixels weighs below $3 / 2$ (expected mean from $[1 / 4,3 / 4)+4$ $* 1 / 4=3 / 2$ ), then label it as a background pixel;
Case 3: if the sum of this pixel and its 4 neighbourhood pixels weighs within $[3 / 2,7 / 2]$, check the sum of this pixel and its 4 diagonal neighbourhood pixels. If that sum weights above 13/4 (lower bound $1 / 4+$ upper bound $4 * 3 / 4$ $=13 / 4$ ), label this pixel as foreground pixel, otherwise background pixel.

Step 4: Classify any other pixel as background pixels.

Note that if the true objects in a dataset are background dominated, we may perform the inverse operation of this labelling policy; if foreground and background objects are equally distributed in a dataset, the aforementioned Steps 13 of this labelling policy, can be adjusted with respect to pixel weights in similar ways. For simplicity of view, considering different cases of possible grayscale histogram distributions, we tabulated this labelling policy referring to the thresholding ches sboard in Table 1.

Table 1. Labelling policy referring to the designed thresholding chessboard

by weighting each point at the intersections on board

\begin{tabular}{|c|c|c|c|c|c|c|c|}
\hline $\begin{array}{l}\text { Threshold } \\
\text { range }\end{array}$ & {$\left[0, \alpha_{1}\right)$} & {$\left[\alpha_{1}, 1 / 4\right)$} & {$\left[1 / 4,1 / 2-\gamma_{1}\right)$} & $\begin{array}{c}{\left[1 / 2-\gamma_{1},\right.} \\
\left.1 / 2+\gamma_{2}\right]\end{array}$ & $\left(1 / 2+\gamma_{2}, 3 / 4\right]$ & $\begin{array}{c}(3 / 4, \\
\left.1-\alpha_{2}\right]\end{array}$ & $\left(1-\alpha_{2}, 1\right]$ \\
\hline $\begin{array}{l}\text { Foreground } \\
\text { dominated }\end{array}$ & 0 & 0 & \multicolumn{3}{|c|}{$\begin{array}{c}\text { Case 1: } 1, \text { if } \operatorname{sum}(p, p 4)>7 / 2 ; \\
\text { Case 2: } 0 \text { if } \operatorname{sum}(\mathrm{p}, p 4)<3 / 2 ; \\
\text { Case 3: } \operatorname{sum}(p, p 4) \in[3 / 2,7 / 2]: 1, \text { if } \operatorname{sum}(p, \operatorname{diag}(p 4))> \\
\text { 13/4; otherwise } 0 .\end{array}$} & 1 & 0 \\
\hline $\begin{array}{c}\text { Background } \\
\text { dominated }\end{array}$ & 0 & 1 & \multicolumn{3}{|c|}{ Inverse results in Cases 1-3 of foreground dominated } & 0 & 0 \\
\hline $\begin{array}{c}\text { Equal } \\
\text { distribution }\end{array}$ & 0 & 1 & \multicolumn{3}{|c|}{$\begin{array}{c}\text { Case } 1: 1, \text { if } \operatorname{sum}(p, p 4)>13 / 4 \\
\text { Case } 2: 0 \text { if } \operatorname{sum}(p, p 4)<7 / 4 ; \\
\text { Case 3: } \operatorname{sum}(p, p 4) \in[7 / 4,13 / 4]: 1, \text { if } \operatorname{sum}(p, \operatorname{diag}(p 4))> \\
\text { 13/4; otherwise } 0 .\end{array}$} & 1 & 0 \\
\hline $\begin{array}{l}\text { Average } \\
\text { concentrate }\end{array}$ & & 0 & $\begin{array}{l}1, \text { if } \operatorname{sum}(p, p 4) \text { and } \\
\operatorname{sum}(p, \operatorname{diag}(p 4)) \\
\operatorname{both} \epsilon[7 / 4,13 / 4] \\
\quad \text { otherwise } 0 \text {. }\end{array}$ & 1 & $\begin{array}{l}1, \text { if } \operatorname{sum}(p, p 4) \text { and } \\
\operatorname{sum}(p, \operatorname{diag}(p 4)) \\
\text { both } \epsilon[7 / 4,13 / 4] \\
\text { otherwise } 0 .\end{array}$ & & ) \\
\hline \multicolumn{8}{|c|}{$\begin{array}{l}\text { Sensitivity Analy sis: with Scaling factors } \alpha_{1}, \alpha_{2}, \gamma_{1}, \gamma_{2}\left(\text { all of } \alpha_{1}, \alpha_{2}, \gamma_{1}, \gamma_{2}<1 / 4\right) \text {; } \\
\text { Shifting factor: } \beta \text {, applied to any } p \text { range } \epsilon\left[\alpha_{1}, 1-\alpha_{2}\right] \text {. } \\
\text { The set of thresholds are adjusted to achieve best overall average F-score. }\end{array}$} \\
\hline
\end{tabular}

We set up $\alpha_{1}=1 / 8$ and $\alpha_{2}=0$. Each algorith $m$ co mbined with the proposed thresholding scheme, has been tested for the two aerial datasets, where the results are discussed in the subsection of 6.4. We also performed a verification of best overall average F-score comparison by adjusting scaling factors and shifting factor before then.

Since even after applying the proposed thresholding scheme, some of the detection errors may still persist, we perform a second stage of post-processing - i.e., an operation of morphological opening to sieve out tiny false detections, followed by a morphological closing operation to connect adjacent small objects, s mooth the border of each detection, and fill the tiny holes inside each detection. We use a structuring element of disk filter with 1-pixel radius to perform all the required opening operations, followed by a $3 \times 3$ structuring element to perform all the closing operations. 


\section{Performance Metrics}

In order to evaluate the performance of each algorithm, we first perform 8-connected component labelling of the binary detection output, then automatically calculate the overlap between detections and ground truth objects. Each type of detections can be classified as follows [13]:

True positive (TP): correct detection. If multiple detections intersect the same ground truth object, then only one TP is counted (the one having largest overlap). If a single detection intersects multiple ground-truth objects, then only one TP is counted (the one having largest overlap);

False negative (FN): detection failure, indicated by a ground truth object failing to intersect any detected object;

False positive (FP): incorrect detection, indicated by a detection that does not intersect any ground truth object;

We use the basic information retrieval (IR) metrics [13], [14] to quantify the detection performance:

Precision: a correctness measure, which is the ratio of TP to the sum of TP and FP;

Recall: a completeness measure, which is the ratio of TP to the sum of TP and FN;

F-score: the harmonic mean of precision and recall;

Percentage of wrong classification (PWC): the ratio of the sum of FP and FN to the sum of TP, FP, FN and TN; since we have no negative samples for detection, $\mathrm{TN}=0$.

The biased F-measure is used as a weighted harmonic mean of precision and recall with a non-negative weight $\beta$, where $\beta^{2}$ is often set as 0.3 for saliency detection [15]:

$$
\mathbf{F}_{\beta}=\frac{\left(1+\beta^{2}\right) \text { Precision } \times \text { Recall }}{\beta^{2} \text { Precision }+ \text { Recall }}
$$

An overlap matrix is generated from detections to groundtruth objects. We denote the areas of object and detection as $M_{i}$ and $A_{j}$, respectively, where each possible touch from detections to objects is expressed via an Ovp matrix, whose element Ovlp $i j$ (measuring percentage of intersection from $i$ th object to $j$-th detection) is calculated as:

$$
\mathrm{Ovlp}_{i j}=\frac{M_{i} \cap A_{j}}{M_{i}+A_{j}-M_{i} \cap A_{j}} \times 100 \%
$$

where $i=1,2, \ldots$, and $j=1,2, \ldots$, co rrespondingly represent the rows and columns of Ovp matrix; we select a threshold ratio $\lambda$ to estimate the overlap ratio between each detection to each object.
While there exists any non-zero element(s) in each row of Ovlp matrix, only label the highest Ovlp $i j$ as TP if it is above $\lambda$, otherwise label it as a FN. We use this metric as a stricter rule when performing sensitivity analysis in the next section.

\section{Experimental Results}

The quantitative detection performance were evaluated in the following scenarios: (1) seven algorithms each combined with the proposed image thresholding scheme, (2) two algorithms displaying best overall performance out of six algorithms combined with this scheme via variable overlap ratios. We also presented a record of average operation time of each algorithm before and after using the proposed twostage scheme.

We adapted five algorithms from previously published research articles, which consist of the Spectrum Residual (SR) model [7], Frequency-Toning (FT) [8], Maximum Symmetric Surroundings (MSS) [9], Laplacian Pyramid Transform (LPT) [10], and Morphological Filtering (MF) [11]. We also modified the algorithm of Multi-scale based Morphological Analysis (MMA) [12] from exploiting multiscale gradient operators to perform the same detection task on two aerial datasets.

\subsection{Six Algorithms each combined with the Proposed Scheme}

We take the first set of experiment to compute the highest average F-score of each algorithm (Alg m.) before and after combining with the proposed post-processing scheme. The average F-score of each algorithm without and with our scheme are displayed in Table 2, where the nu merical results of F-score shows the statistical average of all the tested frames per dataset. In Tucson dataset, LPT with foreground dominated thresholding, achieved the best average F-score (0.897), displaying the most significant improvement to its origin; in Phoenix dataset, MMA with our scheme achieved the highest average F-score (0.727), while LPT with our scheme reached the second highest average F-score of 0.688 ; due to the appearance model diversity and variable degree of motions in different aerial videos, each algorithm combining with the proposed scheme displays better average F-score improvement in Tucson dataset than those in Phoenix dataset. Due to lower resolution and weaker contrast in intensity and enhanced difficulties in feature information of the vehicles, detection performance in Phoenix dataset was worse than that of Tucson dataset when using the same algorithm. 
A Thresholding Scheme of Eliminating False Detections on Vehicles in Wide-Area Aerial Imagery

The classified detections of each algorithm before and after combining the proposed scheme are presented in Table 3 , where the overlap ratio is set as above zero and the last four rows present the quantitative results of average $\mathrm{F}_{\beta}$-score. For each algorithm combining our scheme, the count of FPs is significantly decreased in contrast to its original counts in both datasets, while the removal of FPs results into some loss of TPs for each algorith m; in Tucson dataset, LPT achieves the highest $\mathrm{F}_{\beta}$ score $(0.924)$, all six algorithms achieves average $\mathrm{F}_{\beta}$ score higher than 0.75 ; in Phoenix dataset, MMA displays the highest average $\mathrm{F}_{\beta}$ score (0.718), indicating the only algorith $\mathrm{m}$ with average $\mathrm{F}_{\beta}$ score higher than 0.70 . While the improvements on average $\mathrm{F}_{\beta}$ score suggested uniform discrepancy between the two datasets, even the lowest score (0.494, MF in Phoenix dataset) displayed apparent progress in contrast to its initial detection accuracy.
Table 2. Average F-score for six detection algorithms: before and after combining with the proposed scheme

\begin{tabular}{|c|c|c|c|}
\hline Algm. & F-Score & Tucson (T) & Phoenix (P) \\
\hline \multirow{2}{*}{ SR } & Before & 0.625 & 0.463 \\
\cline { 2 - 4 } & After & 0.765 & 0.589 \\
\hline \multirow{2}{*}{ FT } & Before & 0.555 & 0.389 \\
\cline { 2 - 4 } & After & 0.871 & 0.584 \\
\hline \multirow{2}{*}{ MSS } & Before & 0.510 & 0.412 \\
\cline { 2 - 4 } & After & 0.805 & 0.614 \\
\hline \multirow{2}{*}{ LPT } & Before & 0.243 & 0.199 \\
\cline { 2 - 4 } & After & 0.897 & 0.688 \\
\hline \multirow{2}{*}{ MF } & Before & 0.331 & 0.151 \\
\cline { 2 - 4 } & After & 0.763 & 0.437 \\
\hline \multirow{2}{*}{ MMA } & Before & 0.557 & 0.523 \\
\cline { 2 - 4 } & After & 0.816 & 0.727 \\
\hline
\end{tabular}

Table 3. Classified detections on six algorithms with average F-beta score: before and after applying the proposed thresholding scheme

\begin{tabular}{|c|c|c|c|c|c|c|c|c|}
\hline \multicolumn{3}{|c|}{ Detection Algorithm } & SR & FT & MSS & LPT & MF & MMA \\
\hline \multirow{4}{*}{$\mathbf{T P}$} & \multirow{2}{*}{ Before } & $\mathrm{T}$ & 3575 & 3987 & 3985 & 3894 & 3843 & 3582 \\
\hline & & $\mathrm{P}$ & 2511 & 3253 & 3472 & 2927 & 2910 & 3018 \\
\hline & \multirow{2}{*}{ After } & $\mathrm{T}$ & 3164 & 3529 & 3588 & 3383 & 2603 & 2882 \\
\hline & & $\mathrm{P}$ & 2129 & 2012 & 2318 & 2636 & 1722 & 2475 \\
\hline \multirow{4}{*}{$\mathbf{F N}$} & \multirow{2}{*}{ Before } & $\mathrm{T}$ & 182 & 22 & 12 & 112 & 155 & 235 \\
\hline & & $\mathrm{P}$ & 1097 & 677 & 250 & 815 & 1091 & 587 \\
\hline & \multirow{2}{*}{ After } & $\mathrm{T}$ & 553 & 245 & 201 & 593 & 1329 & 484 \\
\hline & & $\mathrm{P}$ & 1466 & 2000 & 1642 & 971 & 2052 & 844 \\
\hline \multirow{4}{*}{ FP } & \multirow{2}{*}{ Before } & $\mathrm{T}$ & 4259 & 6471 & 7785 & 22387 & 16835 & 5651 \\
\hline & & $\mathrm{P}$ & 3457 & 7550 & 9501 & 22683 & 20907 & 4960 \\
\hline & \multirow{2}{*}{ After } & $\mathrm{T}$ & 239 & 1424 & 1511 & 185 & 241 & 827 \\
\hline & & $\mathrm{P}$ & 1394 & 1345 & 1827 & 1498 & 1677 & 1020 \\
\hline \multirow{4}{*}{$\mathbf{F}_{\boldsymbol{\beta}}$} & \multirow{2}{*}{ Before } & $\mathrm{T}$ & 0.524 & 0.444 & 0.399 & 0.220 & 0.151 & 0.458 \\
\hline & & $\mathrm{P}$ & 0.473 & 0.354 & 0.323 & 0.145 & 0.228 & 0.435 \\
\hline & \multirow{2}{*}{ After } & $\mathrm{T}$ & 0.906 & 0.755 & 0.750 & 0.924 & 0.841 & 0.797 \\
\hline & & $\mathrm{P}$ & 0.610 & 0.574 & 0.569 & 0.674 & 0.494 & 0.718 \\
\hline
\end{tabular}


The IR metrics for each algorithm before and after combining the proposed scheme are presented in Table 4, where the statistical average results on each metric of tested frames in the two aerial datasets are illustrated with sample mean and $95 \%$ confidence interval (CI). Comparing the last column on each dataset in this table, we jus tify that the PWC scores of all six algorithms are reduced to below $50 \%$ in Tucson dataset, while only LPT and MMA improved PWC score to below $50 \%$ in Phoenix dataset. By the proposed scheme, LPT and MMA displays the best two overall PWC improvement among those of six algorithms in our datasets.

\subsection{Best Two Detection Algorithms each Combined with the} Proposed Thresholding Scheme via Variable Overlap Ratios

Two algorithms with the best overall average F-score in two aerial datasets, are selected as the keynote candidates for performance analysis after applying the proposed two-stage scheme over a range of variable overlap ratios. According to the policy of thresholding, classification on each type of detections and average F-score for LPT are presented in Table 5, those of the same metrics for MMA are tabulated in Table 6. It is indicated by the numerical results that MMA exhibits more robust performance in contrast to that of LPT.
Table 4. PWC score of six detection algorithms before and after combining with the proposed thresholding scheme

\begin{tabular}{|c|c|c||c|c|}
\hline \multirow{2}{*}{ Algm. } & \multicolumn{2}{|c||}{ Tucson } & \multicolumn{2}{c|}{ Phoenix } \\
\cline { 2 - 5 } & Before & After & Before & After \\
\hline \multirow{2}{*}{ SR } & $\begin{array}{c}57.90 \\
\pm 0.97\end{array}$ & $\begin{array}{c}21.03 \\
\pm 0.87\end{array}$ & $\begin{array}{c}63.87 \\
\pm 0.94\end{array}$ & $\begin{array}{c}57.05 \\
\pm 0.85\end{array}$ \\
\hline \multirow{2}{*}{ FT } & $\begin{array}{c}61.45 \\
\pm 0.84\end{array}$ & $\begin{array}{c}31.91 \\
\pm 1.12\end{array}$ & $\begin{array}{c}71.54 \\
\pm 0.63\end{array}$ & $\begin{array}{c}62.08 \\
\pm 1.30\end{array}$ \\
\hline \multirow{2}{*}{ MSS } & 65.70 & 32.05 & 73.54 & 59.76 \\
& \pm 0.81 & \pm 1.09 & \pm 0.56 & \pm 0.90 \\
\hline \multirow{3}{*}{ LPT } & 82.15 & $\begin{array}{c}18.66 \\
\pm 1.52\end{array}$ & $\begin{array}{c} \pm 8.83 \\
\pm 0.46\end{array}$ & $\begin{array}{c}47.01 \\
\pm 1.73\end{array}$ \\
\hline \multirow{2}{*}{ MF } & 79.10 & 37.60 & 88.02 & 67.92 \\
& \pm 1.55 & \pm 1.97 & \pm 0.53 & \pm 1.32 \\
\hline \multirow{2}{*}{ MMA } & 61.19 & 30.97 & 64.55 & 42.71 \\
& \pm 1.15 & \pm 1.00 & \pm 0.66 & \pm 0.96 \\
\hline
\end{tabular}

Table 5. Detection performance with updated labeling policy: LPT with the proposed scheme, Tucson and Phoenix dataset (Overlap ratio: $0: 5: 35 \%$ )

\begin{tabular}{|c||c|c|c|c||c|c|c|c||}
\hline \multicolumn{1}{|c||}{ Dataset } & \multicolumn{9}{|c||}{ Tucson } \\
\hline Ovp \% & 0 & 5 & 10 & 15 & 20 & 25 & 30 & 35 \\
\hline TP & 3386 & 3009 & 2428 & 1888 & 1435 & 1059 & 673 & 416 \\
\hline FN & 590 & 967 & 1548 & 2088 & 2541 & 2917 & 3303 & 3560 \\
\hline FP & 182 & 182 & 182 & 182 & 182 & 182 & 182 & 182 \\
\hline F-score & 0.897 & 0.836 & 0.725 & 0.608 & 0.494 & 0.387 & 0.263 & 0.170 \\
\hline Dataset & & & & \multicolumn{1}{|c|}{ Phoenix } & & & \\
\hline Ovp \% & 0 & 5 & 10 & 15 & 20 & 25 & 30 & 35 \\
\hline TP & 2636 & 2191 & 1800 & 1339 & 983 & 688 & 443 & 303 \\
\hline FN & 971 & 1416 & 1807 & 2268 & 2624 & 2919 & 3164 & 3404 \\
\hline FP & 1498 & 1498 & 1498 & 1498 & 1498 & 1498 & 1498 & 1498 \\
\hline F-score & 0.688 & 0.606 & 0.524 & 0.415 & 0.320 & 0.233 & 0.155 & 0.110 \\
\hline
\end{tabular}

Copyright ( 201x Inderscience Enterprises Ltd. 
A Thresholding Scheme of Eliminating False Detections on Vehicles in Wide-Area Aerial Imagery

Table 6. Detection performance with updated labeling policy: MMA with the proposed scheme,

Tucson and Phoenix dataset (Overlap ratio: $0: 5: 35 \%$ )

\begin{tabular}{|c||c|c|c|c||c|c|c|c||}
\hline \multicolumn{1}{|c||}{ Dataset } & \multicolumn{10}{c|}{ Tucson } \\
\hline Ovip \% & 0 & 5 & 10 & 15 & 20 & 25 & 30 & 35 \\
\hline TP & 2879 & 2857 & 2763 & 2594 & 2392 & 2101 & 1814 & 1472 \\
\hline FN & 464 & 486 & 580 & 749 & 951 & 1242 & 1529 & 1871 \\
\hline FP & 887 & 887 & 887 & 887 & 887 & 887 & 887 & 887 \\
\hline F-score & 0.811 & 0.807 & 0.790 & 0.759 & 0.720 & 0.659 & 0.595 & 0.511 \\
\hline Dataset & \multicolumn{7}{|c|}{ Phoenix } \\
\hline Ovlp \% & 0 & 5 & 10 & 15 & 20 & 25 & 30 & 35 \\
\hline TP & 2475 & 2075 & 1846 & 1528 & 1261 & 995 & 774 & 591 \\
\hline FN & 844 & 1244 & 1473 & 1791 & 2058 & 2324 & 2545 & 2730 \\
\hline FP & 1020 & 1020 & 1020 & 1020 & 1020 & 1020 & 1020 & 1020 \\
\hline F-score & 0.727 & 0.643 & 0.592 & 0.514 & 0.442 & 0.364 & 0.294 & 0.231 \\
\hline
\end{tabular}

In more explicit view, we display the performance analysis over a range of variable overlap ratios, where the statistical average of quantitative scores such as precision, recall, F-score and $\mathrm{F}_{\beta}$ score of LPT and MMA over a range of overlap ratios in Tucson dataset, are depicted in a set of subplots in Figure 1. As the overlap ratio increases, LPT
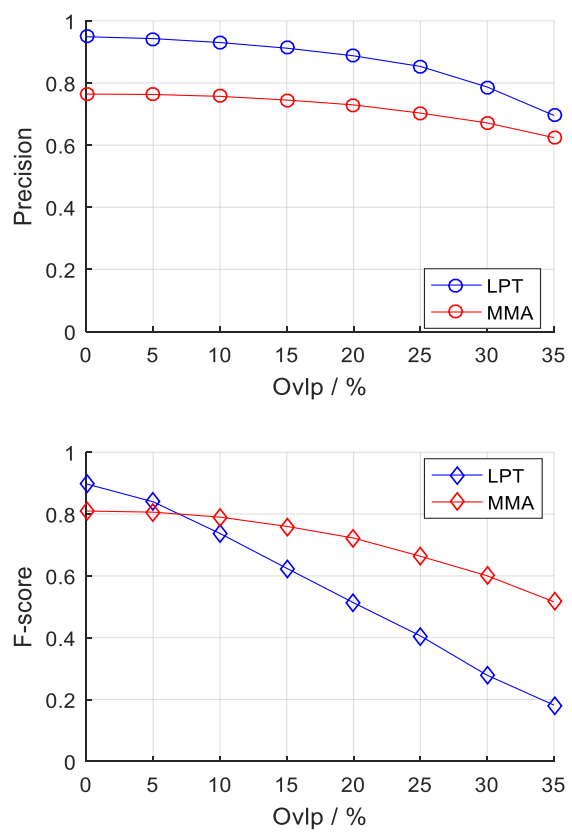

displays better precision but poorer recall comparing to those of MMA, while MMA displays better F-score than LPT in condition that the overlap ratio is higher than $10 \%$; for $\mathrm{F}_{\beta}$ score, MMA performs slightly worse than LPT with overlap ratio lower than $15 \%$, but better than LPT when the overlap ratio ranging from $15 \%$ to $35 \%$.
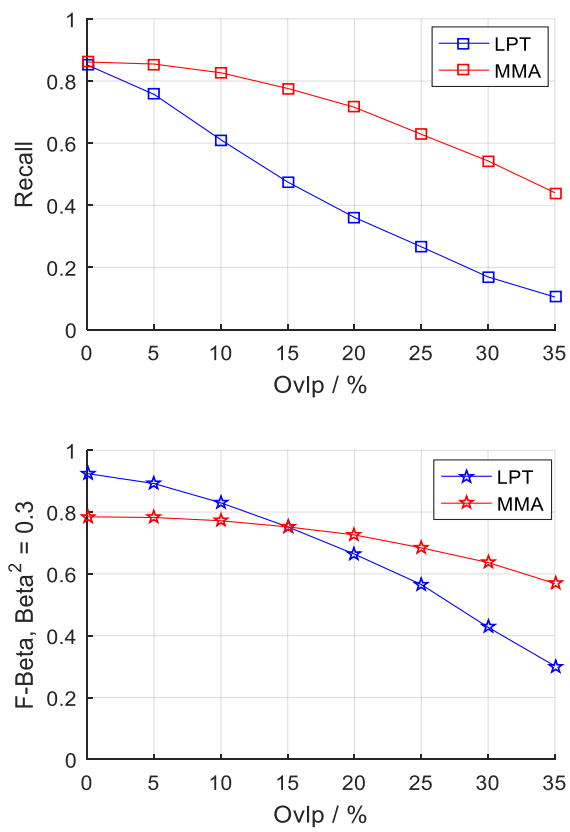

Figure 1. Performance of LPT and MMA with variable quantitative ratio (Overlap $=0 \%: 5 \%: 35 \%$ ), Tucson dataset 
Figure 2 depicts the similar quantitative scores of LPT and MMA over the same range of overlap ratios in Phoenix dataset, where LPT and MMA indicate quite close recall when overlap ratio is no higher than 5\%, while MMA shows higher recall in other cases of overlap ratios. For
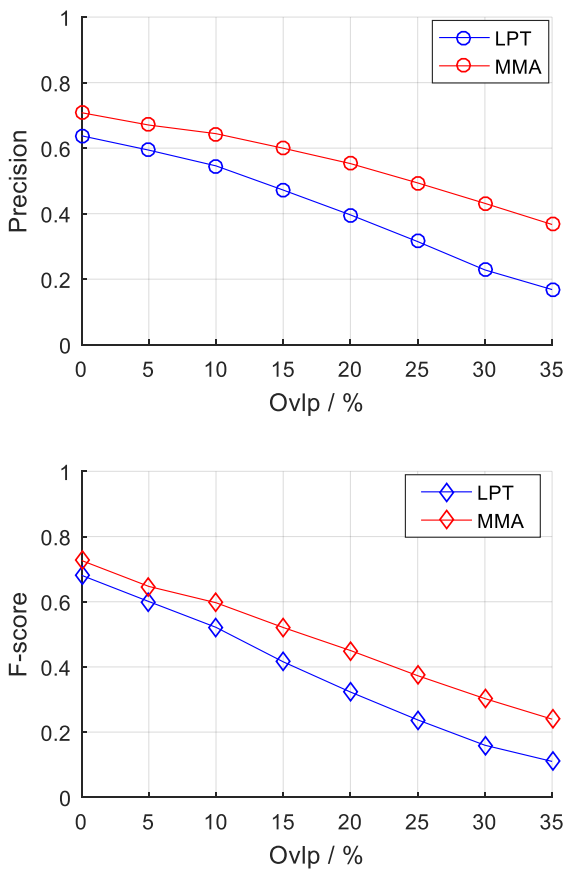

Figure 2. Performance evaluation of LPT and MMA with different quantitative metrics (Overlap ratio $=0: 5: 35 \%)$, Phoenix dataset

\subsection{Average CPU Execution Time}

The last experiment evaluated the average CPU executing time for aerial datasets. We calculated frame-based average operating time on each of the six detection algorithms before and after combining with the proposed thresholding scheme. This test had been conducted in a Toshiba laptop with Windows 10 operation system of Intel Core i7-4510U $2.00 \mathrm{G} \mathrm{CPU}$ and $12 \mathrm{~GB}$ RAM.

The average CPU operation time per frame for each detection algorithm is depicted in Table 7, which was averaged by the total processing time of each algorithm before and after combining our scheme divided by sum of frame numbers on test. Due to the universal efficiency on FP removal, saving computational cost of labelling binary precision, F-score and $\mathrm{F}_{\beta}$ values, MMA uniformly presents better scores than those of LPT. The quantitative measures over variable overlap ratios suggest that MMA indicates comprehensively better overall score, displays more robust performance than those of LPT.
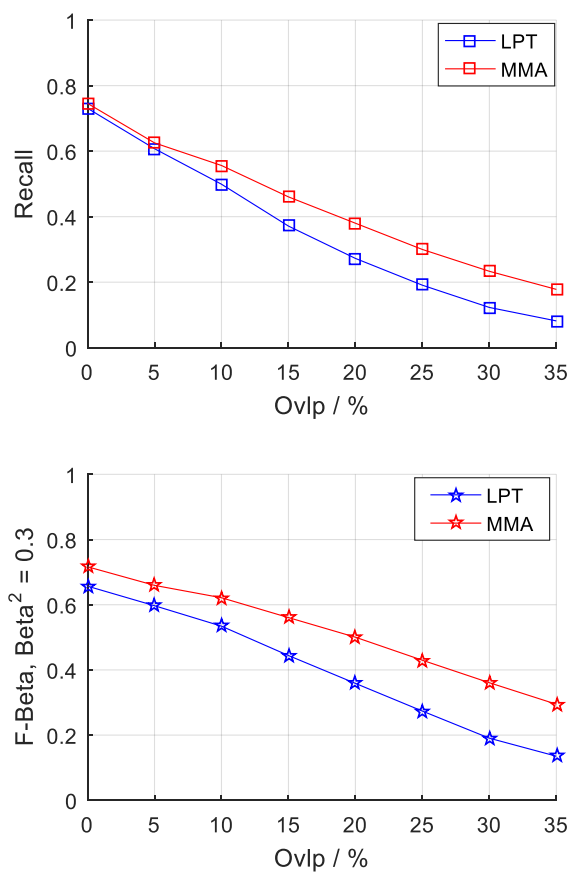

false detections resulted into decreased execution time on flash memory. The average CPU execution time was tested from starting running the first line of MATLAB program till completing classified binary outputs after implementing the proposed scheme. From column comparis on for each algorithm, it is justified that due to its efficiency on reducing FPs, the proposed thresholding scheme is capable of shortening the operation time for each detection algorith m; MF displays the most significant improvement, while LPT shows the least improvement of average CPU execution time. Due to the fact that each of the algorith ms on vehicle detection depends on different technical tools, their corresponding computation costs have the possibility to vary in some certain range of visible effects.

\section{Copyright (C) 201x Inderscience Enterprises Ltd.}


Table 7. Average CPU running time per frame: each detection algorithm without post-processing or combined with the proposed thresholding scheme

\begin{tabular}{|c|c|c|c|c|c|c|}
\hline Before & SR & FT & MSS & LPT & MF & MMA \\
\hline $\begin{array}{c}\text { CPU Time } \\
\text { (seconds) }\end{array}$ & 3.10 & 4.94 & 5.76 & 5.82 & 8.17 & 3.79 \\
\hline After & SR & FT & MSS & LPT & MF & MMA \\
\hline $\begin{array}{c}\text { CPU Time } \\
\text { (seconds) }\end{array}$ & 2.79 & 2.91 & 4.38 & 5.59 & 1.80 & 2.88 \\
\hline
\end{tabular}

\section{Conclusions}

In this paper, we have derived a two-stage post-processing scheme, which comprises a pixel-sum value based image thresholding scheme followed by morphological filtering to eliminate false positives on vehicle detection. The proposed scheme suggests validity and efficiency in improving the detection performance and average CPU execution time on six algorithms for aerial vehicle detection. The labelling policy refers to a thresholding method with respect to several cases of normalized grayscale distributions for segmenting both foreground and background vehicles in different wide-area aerial datasets. The experiments on six detection algorithms combined with the proposed scheme, exhibit improvement for PWC scores on each algorithm. After post-processing with our scheme, LPT displayed an average $\mathrm{F}_{\beta}$ score of above 0.9 , indicating the best result for Tucs on dataset, MMA reached average $\mathrm{F}_{\beta}$ score of above 0.7, ind icating the best result for Phoenix dataset; however, using basic IR metrics for performance evaluation within variable overlap ratios $(0 \%: 5 \%: 35 \%)$ from the classified detections to ground-truth objects, MMA displays better overall quantitative scores than those of LPT.

As future work, we intend to design a motion filter by thresholding the overlap ratio and pixel shift on Euclidean distance among frames after image registration, which will serve as the third step that further eliminates static false detections in order to enhance performance of the proposed scheme. A spatio-temporal post-processing scheme are to be derived in the frequency domain, which is oriented to eliminate false detections in more efficient ways. We also plan to improve the MMA algorithm by exploiting better multi-scale features of aerial vehicles, while verifying its validity in both low-resolution and high-resolution datasets, is supposed to be within our further investigation.

\section{References}

Porter, R., Fraser, A. M. and D. Hush (2010), 'Wide-area motion imagery', IEEE Signal Processing Magazine, Vol. 27, No. 5, pp. 56-65.

Salem, M. A., Ghamry, N. and Meffert B. (2009), 'Daubechies versus biorthogonal wavelets for moving object detection in traffic monitoring systems', Informatik-Berichte 229, ISSN: 0863-095X, pp. 8-9.

Samarabandu J. and Liu, X.-Q. (2007), 'An edge-based text region extraction algorithm for indoor mobile robot navigation', International Journal of Signal Processing, Vol. 3, No. 4, pp. 273-280.

Sharma, B., Katiyar, V. K., Gupta, A. K. and Singh, A. (2014), 'The automated vehicle detection of highway traffic images by differential morphological profile', Journal of Transportation Technology, Vol. 4, pp. 150-156.

Li, S., Zhou, G.-Q., Zheng, Z.-Z., Liu, Y.-L., Li, X.-W., Zhang, Y. and Yue, T. (2013), 'The relation between accuracy and size of structure element for vehicle detection with high resolution highway aerial images', 2013 International Geoscience and Remote Sensing Symposium (IGARSS'2013),pp. 2645-2648.

Gao, X., Ram, S. and Rodríguez, J. J. (2016), 'A performance comparison of automatic detection schemes in wide-area aerial imagery', 2016 IEEE Southwest Symposium in Image Analysis and Interpretation (SSIAI'2016), pp. 125-128.

Hou, X.-D. and Zhang, L.-Q. (2007), 'Saliency detection: A spectral residual approach', Proceedings of IEEE Conference on Computer Vision and Pattern Recognition (CVPR'2007), pp. 1-8. 
X. Gao

Achanta, R., Hemami, S., Estrada, F. and Süsstrunk, S. (2009), 'Frequency-tuned salient region detection', Proceedings of IEEE Conference on Computer Vision and Pattem Recognition (CVPR'2009), pp. 15971604.

Achanta, R. and Süsstrunk, S. (2010), 'Saliency detection using maximum symmetric surround', Proceedings of IEEE Conference on Image Processing (ICIP'2010), pp. 2653-2656.

Unser, M., Chenouard, N. and Ville, D. V. D. (2011), 'Steerable pyramid and tight wavelet frames in $L_{2}\left(\mathbf{R}^{d}\right)^{\prime}$, IEEE Transactions on Image Processing, Vol. 20, No. 10, pp. 2705-2721.

Zheng, Z.-Z., Zhou, G.-Q., Wang, Y., Liu, Y.-L., Li, X.-W., Wang, X.-T. and Jiang, L. (2013), 'A novel vehicle detection method with high resolution highway aerial image', IEEE Journal of Selected Topics in Applied Earth Observations and Remote Sensing, Vol. 6, No. 6, pp. 2338-2343.

Yang, G.-B. and Du. Q.-S. (2010), 'Application and Practical Examples of MATLAB Image / Video Processing', Publishing House of Electronics Library, pp. 149-150.
10

Nascimento, J. C. and Marques, J. S. (2006), 'Performance evaluation of object detection algorithms for video surveillance', IEEE Transactions on Multimedia, Vol. 8, No. 4, pp. 761-774.

Shaikh, S. H., Saeed, K. and Chaki, N. (2014), 'Moving Object Detection Using Background Subtraction', Springer, pp. 30-31.

Borji, A., Cheng, M.-M., Jiang, H.-Z. and Li, J. (2015), 'Salient object detection: a benchmark', IEEE Transactions on Image Processing, Vol. 24, No. 12, pp. 5706-5722.

Moranduzzo, T. (2015), 'DETECTION AND ANALYSIS METHODS FOR UNMANNED AERIAL VEHICLE IMAGES', Ph.D. Dis sertation, University of Trento.

Türmer, S. (2014), 'CAR DETECTION IN LOW-FRAME RATE IMAGERY OF DENSE URBAN AREAS', Ph.D. Dis sertation, Technische University ät München.

Teutsch, M. (2014), 'MOVING OBJECT DETECTION AND SEGMENTATION FOR REMOTE AERIAL VIDEO SURVEILLANCE', Ph.D. Dissertation, Karls ruhe Institute of Technology (KIT), Germany. 